\title{
Assessment of dehiscence and fenestration in children and adolescents using cone beam computed tomography
}

\author{
Aslıhan Akbulut ${ }^{1}$, Beyza Ballı Akgöl ${ }^{2}$, Kaan Orhan $^{3 \dagger}$, Merve Bayram ${ }^{4}$ \\ ${ }^{1}$ PhD. Assistant Professor, Istanbul Medipol University, School of Dentistry, Department of Dentomaxillofacial Radiology, Istanbul, Turkey
}

${ }^{2}$ PhD. Assistant Professor, Istanbul Medipol University, School of Dentistry, Department of Pediatric Dentistry, Istanbul, Turkey

${ }^{3}$ Prof. Dr. Ankara University, School of Dentistry, Department of Dentomaxillofacial Radiology, Ankara, Turkey

${ }^{+}$Ankara University Medical Design Application and Research Center (MEDITAM), Ankara, Turkey

${ }^{4}$ PhD. Assistant Professor, Istanbul Medipol University, School of Dentistry, Department of Pediatric Dentistry, Istanbul, Turkey

\section{Abstract}

Objective: To define the prevalence of dehiscence and fenestration and classify them in terms of the localization of fenestrations in a random sampled group of children and adolescent patients using conebeam computed tomography (CBCT).

Methods: CBCT performed at the Department of Oral and Maxillofacial Radiology of patients referred by the paediatric dentistry clinic were included in this retrospective study. Image evaluations were performed by dentomaxillofacial radiologist (AA, asst. prof.), and these images were examined in three dimensions of the axial, coronal, and sagittal planes. Intraexaminer agreement for the evaluations were found acceptable. The presence/absence of dehiscence and/or fenestration, fenestration's classification type, and localization of defects were recorded. Moreover, the presence/absence of periapical lesion in related root with dehiscence and fenestration was noted. For statistical analysis, The Chi-Square test, Fisher Freeman Halton Test, and Yates' Continuity of Correction were used.

Results: 3061 roots in 1801 teeth of 120 cases were analyzed. The mean age was $9.97 \pm 2.22$ years. Dehiscence was detected in $261(8.5 \%)$ roots of $161(8.9 \%)$ teeth, and fenestration was detected $63(2 \%)$ roots of $36(2 \%)$ teeth. The most common fenestration type was Type I, followed by Type II and IV. Dehiscence was observed more frequently in primary teeth than permanent teeth, and the difference was statistically significant ( $p: 0.000)$. Dehiscence and fenestration incidence in maxillary teeth was significantly higher than in the mandibular teeth $\left(p_{\text {dehiscence }}: 0.000, p_{\text {fenestration }}: 0.004\right)$. Apical lesions were observed more in primary teeth than permanent teeth for both defects.

Conclusion: This study concludes that alveolar dehiscence and fenestrations are more common in primary teeth than permanent teeth. Moreover, these defects were detected more for the teeth in the maxilla. Concerning endodontic and orthodontic therapies in maxilla, use of CBCT is useful in determining the region's anatomical structure accurately in suspected cases of child and adolescent patients.

Keywords: Alveolar bone defect; cone-beam computed tomography; dehiscence; fenestration; primary tooth.

Citation: Akbulut A, et al. (2021) Assessment of dehiscence and fenestration in children and adolescents using cone beam computed tomography. Dentistry 3000. 1:a001 doi:10.5195/d3000.2021.143

Dentistry 3000. 1:a001 doi:10.5195/d3000.2021.143

Received: February 3, 2020

Accepted: April 1, 2021

Published: July 28, 2021

Copyright: (02021 Akbulut A, et al. This is an open access article licensed under a Creative Commons Attribution Work 4.0 United States License.

Email:mbayram@medipol.edu.tr

\section{Introduction}

\section{Diagnosis of alveolar bone defects \\ is crucial for dental practitioners}

due to the fact that the teeth sockets are formed and supported by the alveolar bone. Shape of the defects as well as the position is not predictable and specific to the corresponding region $[1,2]$. The 
Vol 9 No 1 (2021) DOI 10.5195/d3000.2021.143

alveolar bone structure should be correctly assessed for accurate diagnosis and appropriate treatment which affects positive prognosis[1, 3].

Destruction of the alveolar bone, caused by gingival inflammation spreading to the bone, is the leading cause of periodontal disease unless treated properly [4, 5]. This process is exacerbated by anatomical defects in the cortical bone layer that covers the root surfaces [6, 7]. The terms fenestration and dehiscence according to the Glossary of Endodontic Terms are described as follows [8]:

"fenestration - a windowlike opening or defect in the alveolar plate of bone frequently exposing a portion of the root; usually located on the facial aspect of the alveolar process dehiscence - a narrow, vertical defect in the alveolar plate of bone over a root extending from the crestal area apically; usually located on the facial aspect of the alveolar process."

Fenestration and dehiscence have been observed with various reasons such as: i. Endodontic and periapical disease

ii. Trauma

iii. Bruxism

iv. Tooth movement

v. Thin cortical bone

vi. The tooth/jaw ratio

vii. Position of the teeth

viii. Orthodontic problems such as rapid maxillary expansion (RME), orofacial clefts, occlusal forces, posterior crossbite $[2,3,5-7,9-$ 12].

Although some patients experienced a variety of clinical signs and symptoms of fenestration (i.e. persistent pain, discomfort, abscess, and other complex symptoms) [2], others who presented root fenestration did not suffer from any detectable symptoms or discomfort [13]. The outcome of root canal treatment may be affected by preoperative factors such as periapical lesions and periodontal bone defects [14, 15]. When patient suffers from persistent periapical inflammatory symptoms following endodontic treatment, fenestration should be kept in mind as a potentially missing diagnosis [16]. Moreover, dental arch expansion and orthodontic movements of teeth which may cause teeth to move out of the alveolar bone socket leading to dehiscence and fenestration, may be observed primarily in child and adolescent patients. It was indicated that RME may cause fenestration and/or dehiscence in the buccal side of the maxillary teeth [17]. Therefore, detailed imaging is essential to detect problems related to alveolar morphology as it can vary the course of the orthodontic treatment process $[3,10-12,17-$ 21].

Considering the fact that alveolar bone dehiscence is covered with soft tissues; it is invisible to the naked eye and is often difficult to observe clinically. Radiographic assessment is recommended to aid clinical examination for relatively more dependable findings $[1,4,9$, 22-25]. The dehiscence, which occurs on the buccal or lingual side, cannot be observed in routine radiographic diagnosis due to the overlapping images of the surrounding alveolar bone. Therefore, 3D imaging techniques such as cone-beam computed tomography $(\mathrm{CBCT})$ are preferable to $2 \mathrm{D}$ to acquire a better view of surrounding tissues and overcome the issues of superimposition and magnification [9, 17, 19-24, 26]. However, non-essential CBCT use is not recommended for certain patients, in particular children and adolescents, due to the high 
radiation exposure [27]. On the other hand, intraoral radiographs have a sensitivity of $63-67 \%$ in detection and classification of bone defects, whereas CBCT has a reported $[6,22]$ sensitivity of 80 $100 \%$.

Dehiscence and fenestration were observed to have a close relationship with endodontic and/or orthodontic problems and their treatments, as previously mentioned. Therefore, the motivation of this research had been the anticipation of observation of these defects in child and adolescence patients, as well as adults. Previous research in dental literature has predominantly focused on factors affecting the occurrence of dehiscence and fenestration in permanent teeth, and to our knowledge no study has been published evaluating the prevalence of dehiscence and fenestration in primary teeth. The aim of this study was to investigate prevalence of dehiscence and fenestration in children and adolescence, and to classify according to the localization employing CBCT. The study hypothesis was that dehiscence and fenestration prevalence was different in primary teeth than permanent teeth.

\section{Methods}

\section{Study sample}

Ethical approval was obtained from the ethics committee of XXXX University, Clinical Research Ethics Committee, No: XXXXX. The written informed consent forms signed by the parent of the patient as a routine procedure before dental examinations or interventions. Sample size was calculated using the following assumptions; alpha significance level $=0.05$, study power $=80 \%$, anticipated

dehiscence/fenestration prevalence $=10 \%$ based on a previous study [6] and prevalence of study group $=20 \%$. The minimum required sample size was calculated to be 85 cases. CBCT images of patients who applied for dental care in the paediatric clinic referred to the Department of Oral and Maxillofacial Radiology due to $\mathrm{CBCT}$ image requirement for various reasons were evaluated in this retrospective study. The 3D CBCT images were extracted randomly from the archives of the Department of Oral and Maxillofacial Radiology. No CBCT was requested from patients for this study. The patients' demographic data (age, gender, health (systemic disease) status) were recorded.

\section{Inclusion criteria}

In this study setup, three main inclusion criteria were identified to determine the study sample, listed as follows:

i. Child and adolescent patients with $\mathrm{CBCT}$ image.

ii. Complete development of the roots in primary and permanent teeth, observed in CBCT images.

iii. CBCT images with clear visibility of the entire root and surrounding alveolar bone.

\section{Exclusion criteria}

Four main exclusion criteria were identified while determining the study group, listed as follows:

i. Children with malocclusions and/or orthodontic treatment history

ii. Children with systemic disease, syndromes or congenital anomalies

iii. Presence of maxillofacial fracture or trauma history

iv. $\mathrm{CBCT}$ images with obvious pathology (cyst or tumour).

Among the overall identified group 
of 162 patients, the CBCT images of children who had orthodontic treatment history (2 patients), systemic disease (5 patients), congenital anomalies (2 patients), obvious pathology (29 patients), trauma history (4 patients) were excluded from the analysis in the study. A total of 120 CBCT images fulfilled the above-listed inclusion criteria, and were included in the study. All primary and permanent teeth in mandibula and maxilla couldn't be evaluated for each case, because of limited field view based on the reason of image request. Therefore, 578 primary and 1223 permanent teeth were examined in total.

\section{CBCT acquisition and evaluation}

This retrospective study was carried out using the CBCTs taken during the period between July 2011- July 2020. Image evaluations were performed by the same examiner (AA, asst. prof., dentomaxillofacial radiologist, with eighteen years of experience) experienced in CBCT scans, and these images were examined in three dimensions of the axial, coronal, and sagittal planes. For determining the intraexaminer reliability associated with CBCT evaluations, 15 CBCT images were selected randomly, and these corresponding evaluations were repeated two weeks after the first evaluations carried out by the same examiner. Kappa statistics were used to determine intraexaminer agreement for the two sets of evaluations and found to be higher than 0.90 , indicating that all evaluations' reliability was acceptable.

Scans were obtained using CBCT (iCAT ${ }^{\circledR}$, Model 17-19, Imaging Sciences International, Hatfield, $\mathrm{PA}$ ) with a single $360^{\circ}$ rotation and a voxel size of $0.3 \mathrm{~mm}$ with following settings:

i. X-ray emission time was 3.5 seconds

ii. Exposures were made with $5.0 \mathrm{~mA}$, $120 \mathrm{kV}$ and an exposure time of four seconds

iii. The axial slice thickness was 0.3 $\mathrm{mm}$

The images were transformed into data using Invivo 5 Ver. 5.2 Anatomage (San Jose, California, USA) to diagnose the presence of dehiscence and fenestration.

Each root of erupted primary and permanent teeth was evaluated in axial and cross-sectional slices at the buccal and lingual surfaces. An alveolar defect was identified when there was no cortical bone around the root in at least three sequential views. When the alveolar bone height was more than $2 \mathrm{~mm}$. from the cementoenamel junction, it was classified as dehiscence. In addition to this, if the defect did not involve the alveolar crest, the case was classified as fenestration $[2,3,6$, 22 ]. Based on the classification by Pan et al. [9], fenestrations were classified into the following six types (Fig.1): 


\section{Dentistry $3 \square \square \square$}
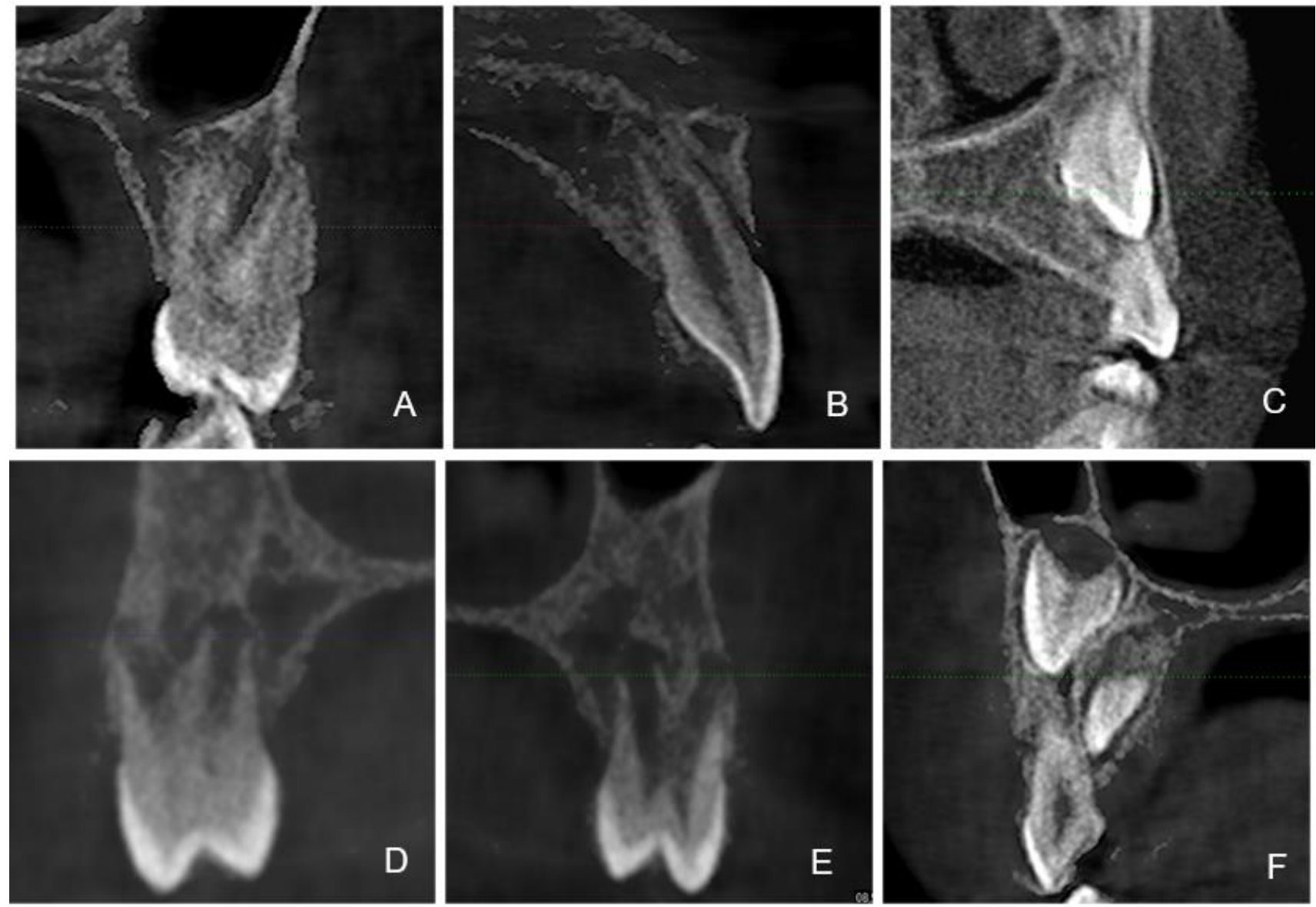

Figure 1. Fenestrations based on the classification by Pan et al. A-Type I; B-Type II; C-Type III; D-Type IV; E-Type V; F-Type VI.

"Type I - protrusion of the apical $1 / 3$ of the root regardless of whether the apical foramen or anatomical apex is involved.

Type II - protrusion of the middle $1 / 3$ of the root.

Type III - protrusion of the coronal $1 / 3$ of the root but excluding the alveolar margin.
Type IV - protrusion of the apical and middle parts of the root regardless of whether the apical foramen or apical apex is involved but excluding the coronal $1 / 3$ of the root.

Type $V$-protrusion of the middle and coronal part of the root without the involvement of the alveolar margin but excluding the apical $1 / 3$ of the root.

Type VI - protrusion of the whole root but excluding the alveolar margin."

\section{Retrospective data acquisition}

Patients were identified by a patient protocol number and case number generated from database records, to protect confidentiality. The case number, age (at the time of the 


\section{口entistry $\exists \square \square \square$}

Vol 9 No 1 (2021) DOI 10.5195/d3000.2021.143

CBCT was taken), gender, health status, tooth number, number of roots for each tooth, presence/absence of a dehiscence (Fig. 2) or fenestration (Fig 3.), fenestration's classification type, and localizations of defects were recorded in a spreadsheet. Moreover, the presence/absence of periapical lesion in related root with dehiscence and fenestration was noted. All data was evaluated by one independent examiner (MB).
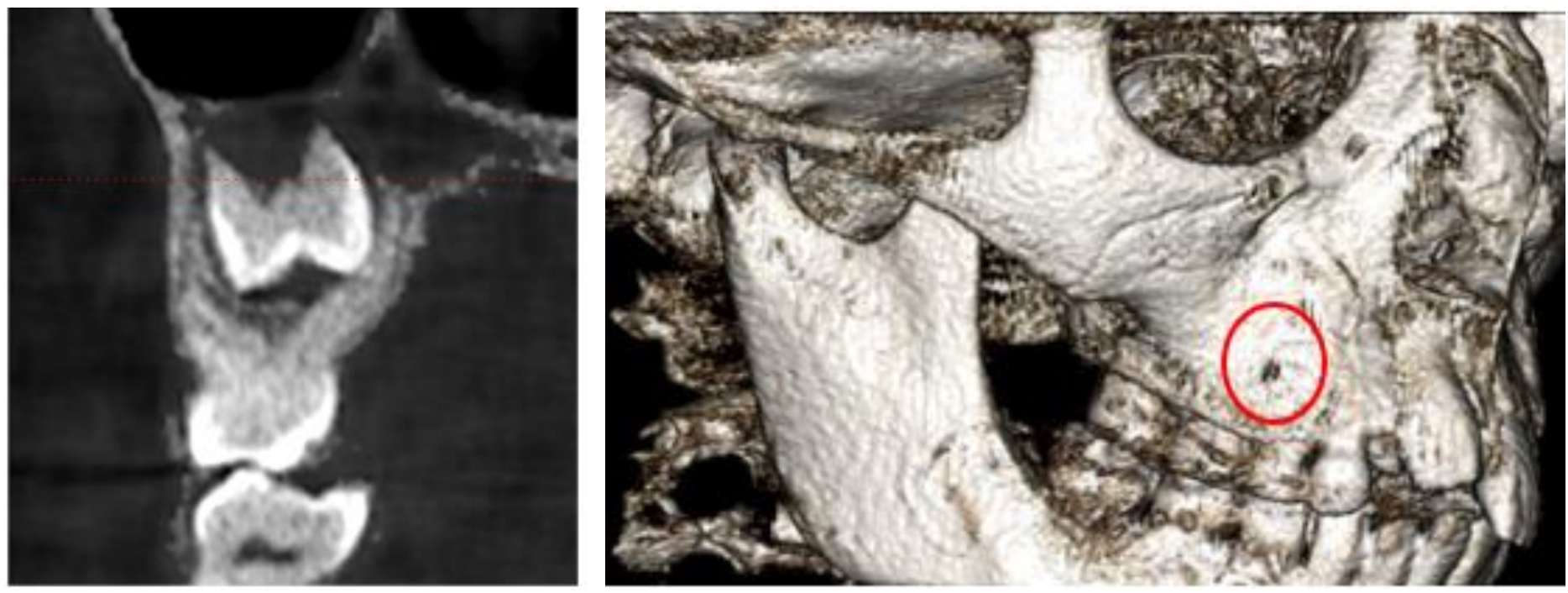

Figure 2. Presence of a dehiscence.

Figure 3. Presence of fenestration - Type I.

\section{Statistical analysis}

Raw patient data extracted from the hospital database was structured using Microsoft Excel (Microsoft Corporation, Redmond, WA, USA) for further analysis. IBM SPSS Statistics 22 for statistical analysis (IBM Corporation, Armonk, NY, USA) was used for statistical analysis. The descriptive statistics (mean, standard deviation, frequency) were recorded. The Chi-Square test, Fisher Freeman Halton Test, and
Yates' Continuity of Correction were used to compare qualitative data. The significance level was set as $<0.05$.

\section{Results}

In this study, 3061 roots in 1801 teeth of 120 cases ( 64 male and 56 female) were evaluated. The ages of the cases ranged between 6 - 15, and the mean age was $9.97 \pm 2.22$ years. Overall prevalence of dehiscence was $40.8 \%$ whereas fenestration was $2.5 \%$. Dehiscence was detected in 261 (8.5\%) roots of 161 (8.9 \%) teeth, and fenestration was detected 63 (2\%) roots of 36 (2 \%) teeth. The presence/absence, and side of dehiscence and fenestrations for concerning root are laid out in Table 1. The dehiscence and fenestration were most frequently observed in the buccal/labial side of the root. The most common fenestration type was Type I (63.9 \%) (Fig. 3), followed by Type II (11.1 \%) and IV (11.1 \%) (Fig. 4) (Table 2). 


\section{Dentistry $30 \square \square$}

Vol 9 No 1 (2021) DOI 10.5195/d3000.2021.143

Table 1. Findings related to dehiscence and fenestration:

Dehiscence $\mathrm{n}(\%) \quad$ Fenestration $\mathrm{n}(\%)$

Defect (teeth; $\mathrm{n}=1801$ )

\begin{tabular}{lll} 
Presence & $161(8.9)$ & $36(2)$ \\
\hline Absence & $1640(91.1)$ & $1765(98)$
\end{tabular}

\section{Defect side}

(teeth; $n_{\text {dehiscence }}=161 n_{\text {fenestration }}=36$ )

\begin{tabular}{lcc} 
Labial/Buccal & $146(90.7)$ & $32(88.9)$ \\
\hline Palatinal/Lingual & $8(5)$ & $4(11.1)$ \\
\hline Labial/Buccal and Palatinal/Lingual & $7(4.3)$ & NA
\end{tabular}

\section{Related root}

(teeth; $n_{\text {dehiscence }}=161 \mathrm{n}_{\text {fenestration }}=36$ )

\begin{tabular}{llc} 
Buccal Root & $4(2.5)$ & $6(16.7)$ \\
\hline Distobuccal Root & $3(1.9)$ & $5(13.9)$ \\
\hline Mesial Root & $3(1.9)$ & NA \\
\hline Mesial Root, Distal Root & $6(3.7)$ & $1(2.8)$ \\
\hline Mesiobuccal Root & $2(1.2)$ & $4(11.1)$ \\
\hline Mesiobuccal Root, Distobuccal Root & $27(16.8)$ & NA \\
\hline Mesiobuccal Root, Distobuccal Root, Palatinal Root & $3(1.9)$ & NA \\
\hline Mesiobuccal Root, Palatinal Root & $4(2.5)$ & NA \\
\hline Palatinal Root & $5(3.1)$ & $19(52.8)$ \\
\hline Single Root & $104(64.6)$ & \\
\hline
\end{tabular}

NA: Non-applicable 


\section{Dentistry $\exists \square \oslash \square$}

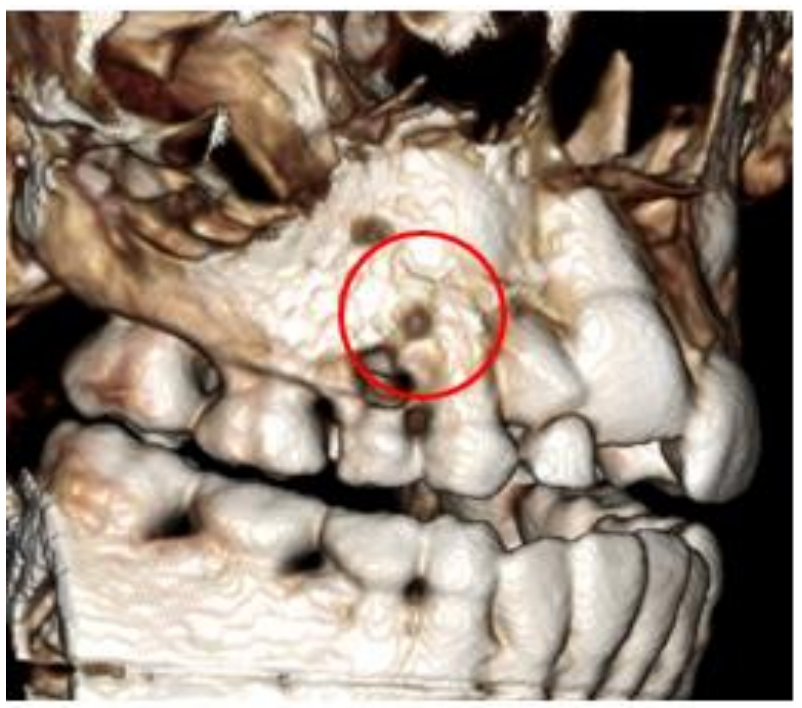

Table 2. Types of fenestration:

\begin{tabular}{lll}
\hline \hline Fenestration types & $\mathbf{n}$ & \% \\
\hline Type I & 23 & 63.9 \\
Type II & 4 & 11.1 \\
Type III & 1 & 2.8 \\
Type IV & 4 & 11.1 \\
Type V & 2 & 5.6 \\
Type VI & 2 & 5.6 \\
\hline \hline
\end{tabular}

Figure 4. Presence of fenestration - Type IV.

The distribution of dehiscence and fenestrations according to the primary/permanent tooth, jaw and tooth type is demonstrated in Table 3. Dehiscence was observed more frequently in primary teeth $(21.5 \%)$ than permanent teeth (3\%). The difference of dehiscence presence between primary and permanent teeth was statistically significant. ( $p: 0.000$, Table 4). Incidence of dehiscence in maxillary teeth (10.9\%) was higher than mandibular teeth (4.6\%) (p:0.000, Table 4). The highest rate of dehiscence was observed in canine (14.9\%) teeth, whereas the lowest rate of dehiscence was noticed in the second premolar (2.7\%). A significant difference for dehiscence frequency was found between tooth types ( $p: 0.000$, Table 4).

Table 3. Frequency of dehiscence / fenestration, based on tooth type:

\begin{tabular}{|c|c|c|c|c|}
\hline \multirow{2}{*}{ Primary/permanent } & \multirow{2}{*}{ Jaw } & \multirow{2}{*}{ Tooth Type } & \multirow{2}{*}{$\begin{array}{l}\text { Dehiscence } \\
\text { n (\%) }\end{array}$} & \multirow{2}{*}{$\begin{array}{l}\text { Fenestration } \\
\mathbf{n}(\%)\end{array}$} \\
\hline & & & & \\
\hline \multirow[t]{10}{*}{ Permanent Tooth } & Maxilla & Incisor & $14(4.5)$ & $12(3.8)$ \\
\hline & & Canine & $3(5.2)$ & $0(0)$ \\
\hline & & First premolar & $4(5)$ & $5(6.3)$ \\
\hline & & Second premolar & $1(1.6)$ & $2(3.2)$ \\
\hline & & First molar & $2(1)$ & $0(0)$ \\
\hline & & Second molar & $3(7.1)$ & $0(0)$ \\
\hline & Mandible & Incisor & $3(1.7)$ & $0(0)$ \\
\hline & & Canine & $2(3.9)$ & $0(0)$ \\
\hline & & First premolar & $2(3.4)$ & $0(0)$ \\
\hline & & Second premolar & $2(4.1)$ & $0(0)$ \\
\hline
\end{tabular}


Assessment of dehiscence and fenestration in children and adolescents using cone beam computed tomography

\section{Dentistry $30 \square \square$}

Vol 9 No 1 (2021) DOI 10.5195/d3000.2021.143

\begin{tabular}{|c|c|c|c|c|}
\hline & & First molar & $1(1.1)$ & $0(0)$ \\
\hline & & Second molar & $0(0)$ & $0(0)$ \\
\hline \multirow[t]{8}{*}{ Primary tooth } & Maxilla & Incisor & $36(50)$ & $2(2.8)$ \\
\hline & & Canine & 35 (23.5) & $2(1.3)$ \\
\hline & & First molar & 21 (17.6) & $8(6.7)$ \\
\hline & & Second molar & 16 (11.9) & $2(1.5)$ \\
\hline & Mandible & Incisor & $2(28.6)$ & $0(0)$ \\
\hline & & Canine & 4 (10.8) & $2(5.4)$ \\
\hline & & First molar & $5(19.2)$ & $1(3.8)$ \\
\hline & & Second molar & $5(14.7)$ & $0(0)$ \\
\hline
\end{tabular}

Table 4. Tooth and jaw relations with dehiscence and fenestration:

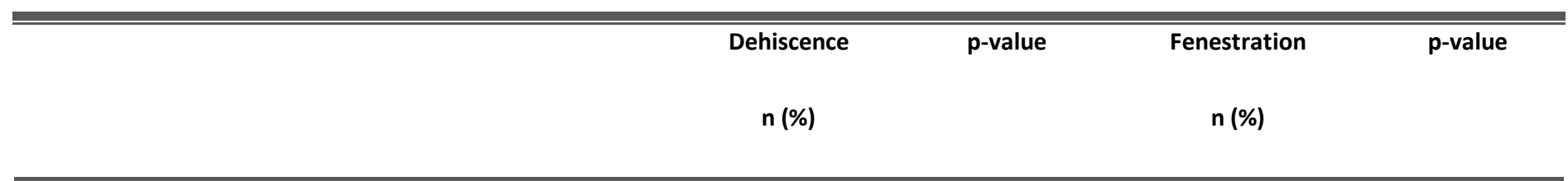

\section{Primary/permanent}

\begin{tabular}{|c|c|c|}
\hline \multirow[t]{2}{*}{ Permanent teeth } & $37(3)$ & $19(1.6)$ \\
\hline & & \\
\hline Primary teeth & $124(21.5)$ & $17(2.9)$ \\
\hline
\end{tabular}

${ }^{\ddagger} 0.074$

$\begin{array}{lll}\text { Primary teeth } & 124(21.5) & 17(2.9)\end{array}$

\begin{tabular}{|c|c|c|c|c|c|}
\hline \multicolumn{6}{|l|}{ Jaw } \\
\hline & Maxilla & 135 (10.9) & & $33(2.7)$ & \\
\hline & & & +0.000 & & ${ }_{0.004}$ \\
\hline & Mandible & $26(4.6)$ & & $3(0.5)$ & \\
\hline \multicolumn{6}{|l|}{ Tooth type } \\
\hline & Incisor & $55(9.6)$ & & $14(2.4)$ & \\
\hline & Canine & 44 (14.9) & & $4(1.4)$ & \\
\hline & First premolar & $6(4.3)$ & & $5(3.6)$ & \\
\hline & & & +0.000 & & $\S 0.437$ \\
\hline & Second premolar & $3(2.7)$ & & $2(1.8)$ & \\
\hline & First molar & $29(6.6)$ & & $9(2.1)$ & \\
\hline & Second molar & $24(9.8)$ & & $2(0.8)$ & \\
\hline
\end{tabular}




\section{Dentistry $\exists \square \oslash 0$}

Vol 9 No 1 (2021) DOI 10.5195/d3000.2021.143

+ Chi-square test

'Yates' Continuity of Correction \$Fisher

Freeman Halton Test

Table 5. Distribution of periapical lesion in teeth with dehiscence and fenestration:

\begin{tabular}{c|c}
\hline \hline & Dehiscence \\
& $\mathbf{n}(\%)$ \\
\hline \hline Total & $161(8.9)$ \\
\hline Periapical lesion & $32(19.8)$ \\
\hline Primary teeth & $28(87.5)$ \\
\hline Permanent teeth & $4(12.5)$ \\
\hline
\end{tabular}

Dehiscence of different tooth types was demonstrated in Fig. 5. Fenestration was observed in $1.6 \%$ of permanent teeth and $2.9 \%$ of primary teeth, the difference was not statistically significant $(p>0.05$, Table 4). Fenestrations were more frequent in maxillary teeth $(2.7 \%)$ than in mandibular teeth $(0.5 \%)$. Fenestration was not observed in any permanent mandibular teeth. There was a significant difference for fenestration frequency between the lower and upper teeth ( $p: 0.004$, Table 3). The highest incidence of fenestration was observed in the first premolar (3.6\%) teeth, whereas the lowest incidence of teeth with fenestration was in the second molar (0.8 \%). There was no statistically significant difference between fenestration incidence

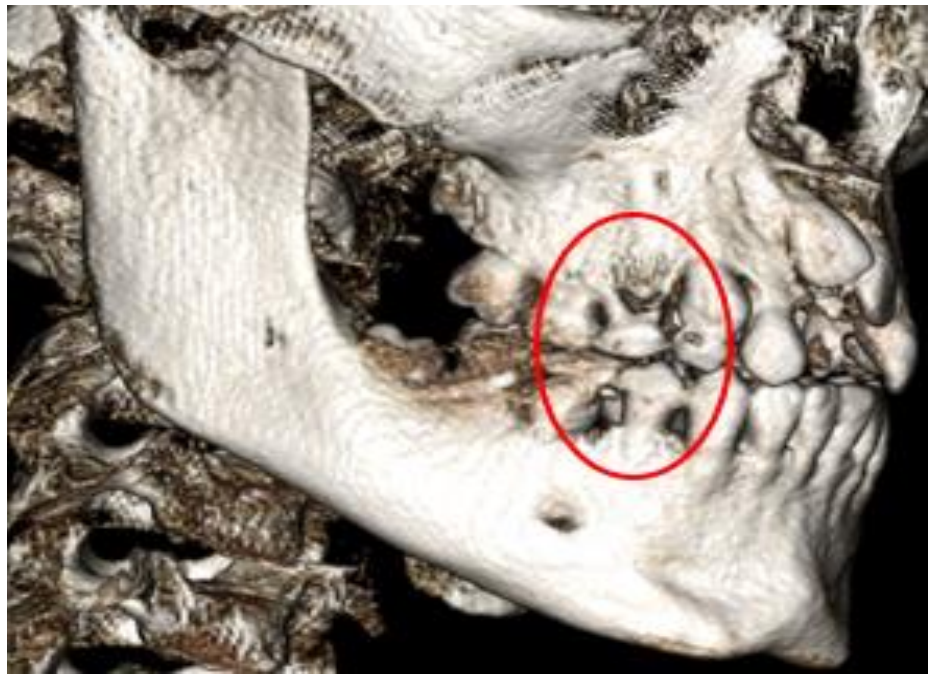

Figure 5. Dehiscence of different tooth types.

according to tooth types ( $p>0.05$, Table 4). Table 5 lays out the number and frequency of apical lesion presence in teeth with seen dehiscence and fenestration.

The periapical lesions were observed more frequently in teeth with dehiscence $19.8 \%$ than with fenestration $5.5 \%$. Periapical lesions were observed more in primary teeth than permanent teeth for both defects.

\section{Discussion}

In this retrospective study 1801 teeth were inspected for dehiscence and fenestration defects in child and adolescent 
patients. Moreover, fenestration classification types were evaluated. To our knowledge, no previous studies have analyzed the prevalence of dehiscence and fenestration in primary teeth.

The limitations of this study are as follows:

i. Thickness of the cortical bone may be influenced from hormonal and functional changes which is associated with the study group. The age range was between $6-15$ years, and this study group contained growing patients.

ii. All teeth in both jaws couldn't be evaluated for each case due to the retrospective acquisition of $\mathrm{CBCT}$ records, which included limited field view based on the reason of image request.

Among the 1801 teeth evaluated, (8.9\%) had alveolar dehiscence. This result was higher than the previous reported dry human skulls investigation studies $(3.2 \%$ $7.1 \%)$, yet was similar to a previous CBCT study (8.6 \%). The current study showed that dehiscence (8.9 \%) was seen more frequently than fenestration (2\%), which is in line with previous researches [10, $11,19,21] .90 .7 \%$ of the dehiscence was on the buccal/labial side while $5 \%$ were on the lingual/palatinal side, and these findings are consistent with previous studies $[9,12,18,19]$. The highest rate of dehiscence was seen in single root teeth (64.6\%), followed by mesiobuccal and distobuccal roots $(16.8 \%)$. On the other hand, dehiscence, only involving the mesiobuccal root was $1.2 \%$.

In the past, fenestration and dehiscence in dry human skulls and flap surgery on cadaver heads were used merely to investigate the prevalence. Until the introduction of computer tomography (CT), visualization of labial/buccal and lingual bone plates was not possible because of the image superimposition associated with conventional radiographs [10]. 2D radiographic imaging is not sufficient to show the alveolar bone defects, especially in the posterior area. When significant buccolingual tooth movement or tipping is required during orthodontic treatment, it becomes necessary to be aware of existing bone defects in the alveolar area which may make complex the orthodontic treatment process [28]. CBCT has provided to visualize these defects three-dimensionally
[11]. CT images can now show bone dehiscence and fenestration utilizing high definition and sensitivity.[18] CBCT also yields high-definition images of teeth and bone at a far lower dosage of radiation that was formerly used with medical imaging and closer to the range of standard dental film series [12]. As can be seen in various animal experiments, the loss of thin bone plates may be induced by orthodontic tooth movement [17]. Therefore, the treatment plan's choice should depend on bone morphology and the position of the teeth $[19,20]$. Imaging anatomic details of the patients and comprehension of tooth movement collateral effects allow us to safely realize the borders and practice, unlike the traditional method of investigating the prevalence and morphology of fenestration on dry human skulls.

Visual examination and direct measurement made the traditional methods using dry human skulls are highly accurate and reliable despite its disadvantages as dried skulls studies offer no clinical information. Moreover, this method could never be applied to clinical endodontic diagnosis. However, it must be taken into consideration that both in vivo and ex vivo studies have indicated that CBCT is likely to be a useful and 
more practical clinical tool than digital subtraction radiography or $\mathrm{CT}$, for almost all endodontic and periodontal periapical applications [29]. In this respect, the low doses of radiation and superior image quality of CBCT compared with conventional CT were worthwhile in diagnosing dehiscence and fenestration defects [30].

Following the proper root canal treatment, teeth are generally asymptomatic; the pain symptoms are not associated with bone defect. However, mechanical and chemical irritation caused by contact of the periosteum and mucosa with foreign material after overflowing root canal filling may cause temporary or permanent pain. In the presence of dehiscence, direct contact of the canal filling material with the mucosa may cause spontaneous pain. Apical fenestration may be considered an anatomic predisposing factor for persistent pain following root canal treatment which often is misdiagnosed [31]. When planning for a root canal treatment, a careful examination should be carried out not only for the root but also the tissues around the root. Dehiscence and fenestration may cause ongoing pain, unsuccessful treatment outcome accompanied by periodontal problems, especially following endodontic treatment. Therefore, the possibility of apical fenestration should be precisely considered with $\mathrm{CBCT}$ image when necessary. In the current study, addition to prevalence of dehiscence and fenestration, periapical lesions were detected in these teeth with dehiscence and fenestrations defects, leading to pathological changes.

Dehiscence and fenestration were more frequently observed in maxillary teeth than in mandibular teeth in this research which is in line with previous studies $[5,6,9$, 12, 17, 18]. Although this phenomenon has not been understood yet, Nimigean et al. [2] has hypothesized that potential reason for seeing more fenestrations in the maxillary arch is root tip inclination to the labial/buccal for teeth in maxilla. The current study strengthens the previous findings $[6,7,9,12,18$, 19] reporting far more frequent dehiscence and fenestration on the labial/buccal side, than on the palatal/lingual.

In a previous study, frequency of permanent tooth types with dehiscence was found highest in maxillary first permanent molars and least in mandibular permanent molars. The present study demonstrated that maxillary permanent canine has the highest dehiscence frequency, where mandibular permanent molars have the lowest. Ectopic eruption may be considered a potential reason for the high observation frequency in the upper canine tooth. The study group was mostly in the mixed dentition stage, and ectopic eruption was clearly one of the aetiological factors that affects bone volume housing the teeth [32].

In the maxilla, frequency of permanent tooth types with fenestration was higher in premolar teeth followed by incisors. In addition to this, fenestration was not observed in any permanent tooth type in mandible. Previous researchers [2, 33] reported different pattern for relative fenestration frequency in permanent tooth types as follows: maxillary first molar, maxillary/mandibular canines and mandibular lateral incisors. The difference is probably due to the fact that the above-mentioned researches could make an assessment including all permanent teeth. Considering our study group which included patients in mixed dentition stage, it was not possible to evaluate all permanent teeth. 
There is a tendency to perceive fenestration as a rare condition [9] and there exists a few case reports, $[13,16,31,34]$ among which only one related with children. The frequency of root fenestrations in this study was found low (2\%), and type I fenestrations was the highest proportion (63.9 \%) among all types. Type of fenestration findings in the current study was in line with the Chinese subpopulation study [9]. In this study, $5.5 \%$ of teeth with fenestration had periapical lesions, all of which was associated with primary teeth. Besides, the presence of periapical lesions was found to be higher in teeth with dehiscence compared to with fenestration.

Alveolar bone loss caused by periapical lesions may also be related to dehiscence and fenestration. During the pretreatment period, clinicians are generally focused on the clinical status of supporting tissues and implications on treatment. Treatment plan gets complicated by the complex modelling of the alveolus in a growing patient by growth rotations of the skeletal structures that affect the direction of compensatory eruptive movements.
This study concludes that alveolar dehiscence and fenestration defects are more common in primary teeth than permanent teeth. Moreover, the teeth in the maxilla were identified as highly affected from these defects. Concerning endodontic and orthodontic therapies in maxilla, use of CBCT is useful in determining the region's anatomical structure accurately in suspected cases of child and adolescent patients. Persistent pain following root canal treatment in children should be further investigated for possible dehiscence and fenestration presence. This study's clinical relevance is providing the result of high defect prevalence in primary teeth, to assist dental professionals perceive which teeth are most often associated with alveolar defects, which may be considered on the diagnosis and treatment plans.

Conflict of Interest: The authors declare that they have no conflict of interest.

Funding: The authors declare that this study has received no financial support.

Ethical approval: Ethics committee approval was received for this study from the Ethics Committee of Istanbul Medipol University (No: 10840098-604.01.01-E.6363)

\section{Author contributions:}

KO conceptualized the manuscript. BBA and AA carried out methodology; MB carried out data analysis, drafted and edited the manuscript. All authors subsequently revised the draft. All authors read and approved the final manuscript.

\section{References}

1. Comparison of linear and volumetric measurements obtained from periodontal defects by using cone beam-CT and micro-CT: an in vitro study. Tayman MA, Kamburoğlu K, Küçük Ö, et al (2019). Clin Oral Investig 23:2235-2244. PMID: 30284102

2. Alveolar bone dehiscences and fenestrations: An anatomical study and review. Nimigean VR, Nimigean $\mathrm{V}$, Bencze MA, et al (2008). Rom J Morphol Embryol 50:391-397. PMID: 19690764

3. Accuracy of cone-beam computed tomography in detecting alveolar bone dehiscences and fenestrations. Sun L, Zhang L, Shen G, et al (2015). Am J Orthod Dentofac Orthop 147:313-323. PMID: 
Vol 9 No 1 (2021) DOI 10.5195/d3000.2021.143

\section{8}

4. Classification of alveolar bone destruction patterns on maxillary molars by using cone-beam computed tomography. Ozcan G, Sekerci AE (2017). Niger J Clin Pract 20:1010-1019.

PMID: 28891547

5. Cone-Beam Computed Tomography Evaluation of Alveolar Bone Dehiscences on Mesiobuccal Root Region of Upper First Molar Teeth. Nalcı G, Alaçam T, Salmanov E, et al (2020). Turkiye Klin J Dent Sci 26:195-200

6. KKTC toplumunda fenestrasyon ve dehisens prevalansının konik ışınlı bilgisayarlı tomografi ile değerlendirilmesi. Aksoy U, Aksoy S (2019). 317-322

7. Alveolar bone fenestrations and dehiscences in dry Bedouin jaws. Edel A (1981). J Clin Periodontol 8:491-499. PMID: 6949922

8. Glossary of Endodontic Terms. American Association of Endodontists (2015). Gloss Endod Terms

\section{9:43}

9. Use of cone-beam computed tomography to evaluate the prevalence of root fenestration in a Chinese subpopulation. Pan $H Y$, Yang $H$, Zhang R, et al (2014). Int Endod J 47:1019. PMID: 23701176

10. Dehiscence and fenestration in skeletal Class I, II, and III malocclusions assessed with cone-beam computed tomography. Yagci A, Veli I, Uysal T, et al (2012). Angle Orthod 82:67-74. PMID: 21696298

11. Dehiscence and fenestration in patients with different vertical growth patterns assessed with cone-beam computed tomography. Enhos S, Uysal $T$, Yagci A, et al (2012). Angle Orthod 82:868-874. PMID: 22356702

12. Appraisal of the relationship between tooth inclination, dehiscence, fenestration, and sagittal skeletal pattern with cone beam computed tomography. Coskun I, Kaya B (2019). Angle Orthod 89:544-551. PMID: 30741575

13. Management of mucosal fenestrations by interdisciplinary approach: a report of three cases. Jhaveri HM, Amberkar S, Galav L, et al (2010). J Endod 36:164-168. PMID: 20003959

14. Outcome of secondary root canal treatment: a systematic review of the literature. Ng Y-L, Mann V, Gulabivala K (2008). Int Endod J 41:1026-1046. PMID: 19133093

15. A prospective study of the factors affecting outcomes of nonsurgical root canal treatment: part 1: periapical health. Ng Y-L, Mann V, Gulabivala K (2011). Int Endod J 44:583-609. PMID: 21366626

16. Persistent pain related to root canal filling and apical fenestration: a case report. Boucher $Y$, Sobel M, Sauveur G (2000). J Endod 26:242-244. PMID: 11199728

17. Evaluation of alveolar bone loss following rapid maxillary expansion using cone-beam computed tomography. Baysal A, Uysal T, Veli I, et al (2013). Korean J Orthod 43:83-95. PMID: 23671833 
18. Detection of Bone Defects Using CBCT Exam in an Italian Population. Gambarini G, Miccoli G, Gaimari G, et al (2017). Int J Dent 2017:

19. Prevalence of posterior alveolar bony dehiscence and fenestration in adults with posterior crossbite: a CBCT study. Choi JY, Chaudhry K, Parks E, Ahn JH (2020). Prog Orthod 21:. PMID: 32173764

20. Evaluation of dehiscence and fenestration in adolescent patients affected by unilateral cleft lip and palate: A retrospective cone beam computed tomography study. Buyuk SK, Ercan E, Celikoglu M, et al (2016). Angle Orthod 86:431-436.

PMID: 26284755

21. Dehiscence and fenestration in patients with class i and class II division 1 malocclusion assessed with cone-beam computed tomography. Evangelista K, Vasconcelos KDF, Bumann A, et al (2010). Am J Orthod Dentofac Orthop 138:133.e1-133.e7. PMID: 20691344
22. Comparison of the influence of FOV sizes and different voxel resolutions for the assessment of periodontal defects. Kolsuz ME, Bagis N, Orhan K, et al (2015). Dentomaxillofacial Radiol 44:. PMID: 25900235

23. Detection of simulated periodontal defects using cone-beam CT and digital intraoral radiography. Bayat S, Talaeipour AR, Sarlati F (2016). Dentomaxillofacial Radiol 45:

24. Diagnostic accuracy of CBCT for periodontal lesions. Braun X, Ritter L, JervøeStorm PM, Frentzen M (2014). Clin Oral Investig 18:1229-1236. PMID: 24048949

25. Comparison of intraoral radiography and cone-beam computed tomography for the detection of periodontal defects: An in vitro study. Bagis N, Kolsuz ME, Kursun S, Orhan K (2015). BMC Oral Health 15:1-8. PMID: 26016804

26. Diagnostic validity (or acuity) of 2D CCD versus 3D CBCT-images for assessing periodontal breakdown. Vandenberghe $B$, Jacobs $R$,
Yang J (2007). Oral Surgery, Oral Med Oral Pathol Oral Radiol Endodontology 104:395-401. PMID: 17613257

27. Indications for cone beam computed tomography in children and young patients in a Turkish subpopulation. İşman Ö, Yılmaz HH, Aktan AM, Yilmaz B (2017). Int J Paediatr Dent 27:183-190. PMID: 27452447

28. Changes of alveolar bone dehiscence and fenestration after augmented corticotomy-assisted orthodontic treatment: a CBCT evaluation. Sun $\mathrm{L}$, Yuan L, Wang B, et al (2019). Prog Orthod 20: PMID: 30773604

29. Cone-Beam CT Diagnostic Applications: Caries, Periodontal Bone

Assessment, and Endodontic Applications. Tyndall DA, Rathore S (2008). Dent Clin North Am 52:825-841. PMID: 18805231

30. Digital volume tomography for diagnostics in periodontology. Kasaj $A$, Willershausen B (2007). Int J Comput Dent 10:155-168. 
Vol 9 No 1 (2021) DOI 10.5195/d3000.2021.143

PMID: 17899891

31. Atypical facial pain related to apical fenestration and overfilling. Pasqualini D, Scotti N, Ambrogio P, et al (2012). Int Endod J 45:670677. PMID: 22309707

32. Pattern of bone resorption in vertically fractured, endodontically treated teeth. Lustig JP, Tamse A, Fuss Z (2000). Oral Surg Oral Med Oral Pathol Oral Radiol Endod 90:224-227. PMID: 10936842

33. Prevalence of Dehiscences and Fenestrations in Modern American Skulls. Rupprecht RD, Horning GM, Nicoll BK, Cohen ME (2001). J Periodontol 72:722-729.

PMID: 11453233

34. The management of mucosal fenestration: a report of two cases. Chen $\mathrm{G}$, Fang CT, Tong C (2009). Int Endod J 42:156-164. PMID: 19134044 